\title{
MAXIMUM AVERAGE DISTANCE IN COMPLEX FINITE DIMENSIONAL NORMED SPACES
}

\author{
Juan C. García-Vázquez and Rafael Villa
}

\begin{abstract}
A number $r>0$ is called a rendezvous number for a metric space $(M, d)$ if for any $n \in \mathbb{N}$ and any $x_{1}, \ldots x_{n} \in M$, there exists $x \in M$ such that $(1 / n) \sum_{i=1}^{n} d\left(x_{i}, x\right)=r$. A rendezvous number for a normed space $X$ is a rendezvous number for its unit sphere. A surprising theorem due to $O$. Gross states that every finite dimensional normed space has one and only one average number, denoted by $r(X)$. In a recent paper, A. Hinrichs solves a conjecture raised by $\mathrm{R}$. Wolf. He proves that $r(X) \leqslant r\left(\ell_{1}^{n}\right)=2-1 / n$ for any $n$-dimensional real normed space. In this paper, we prove the analogous inequality in the complex case for $n \geqslant 3$.
\end{abstract}

\section{INTRODUCTION}

A number $r>0$ is called a rendezvous number for a metric space $(M, d)$ if for any $n \in \mathbb{N}$ and any $x_{1}, \ldots x_{n} \in M$, there exists $x \in M$ such that

$$
\frac{1}{n} \sum_{i=1}^{n} d\left(x_{i}, x\right)=r
$$

In 1964, Gross [4] proved that every compact connected metric space has one and only one rendezvous number. In this case, the unique rendezvous number is denoted by $r(M, d)$, and it is said that $(M, d)$ has the average distance property. The general inequalities $D / 2 \leqslant r \leqslant D$ can be easily checked for any rendezvous number $r$ of a metric space with diameter $D$. Moreover, for a compact metric space, the second inequality is $r<D$ (see [10]).

Consider a normed space $X$. It is known (see [2] or [13]) that the unit ball of $X$ has the average distance property, with 1 as the unique rendezvous number. A much more interesting case is the unit sphere $S(X)$ of $X$. If $X$ is a finite dimensional normed space, a direct application of Gross's theorem implies that $S(X)$ has the average distance property, and its rendezvous number, called the rendezvous number of $X$, is

Received 5th February, 2002

Research supported by DGES grant \#BFM2000-0514.

Copyright Clearance Centre, Inc. Serial-fee code: 0004-9727/02 \$A2.00+0.00. 
denoted simply by $r(X)$. In this case we also know that $1<r(X)<2$ (see [15]). Calculations for these numbers in classical spaces have been carried out:

(1) $r\left(\ell_{1}^{n}\right)=2-1 / n ; r\left(\ell_{\infty}^{n}\right)=3 / 2($ see $[13])$

(2) $r\left(\ell_{2}^{n}\right)=\left(2^{n-2} \Gamma(n / 2)^{2}\right) /(\sqrt{\pi} \Gamma(2 n-1 / 2))(\rightarrow \sqrt{2}$ as $n \rightarrow \infty)$ (see [8])

(3) $\quad r\left(\ell_{p}^{n}\right) \rightarrow 2^{1 / p}$ as $n \rightarrow \infty$ (see [7])

Since $X \mapsto r(X)$ is continuous on the Minkowski compactum of normed spaces of fixed dimension $n$ (see [1]), it follows that there is an $n$-dimensional normed space $X_{0}$ such that $r(X) \leqslant r\left(X_{0}\right)<2$ for any $n$-dimensional normed space $X$. It was conjectured by Wolf in [13] that the maximum, for $n \geqslant 2$, is attained for $\ell_{1}^{n}$. Thus the conjecture can be written

$$
r(X) \leqslant 2-\frac{1}{n}
$$

for any $n$-dimensional normed space $X$. The same author proved the inequality for $n=2[13]$, for any $X$ with a 1-unconditional basis ([14]) and for any $X$ isometrically isomorphic to a subspace of $L^{1}[0,1]([17])$. Moreover, he proved that equality holds in these three cases if and only if $X$ is isometrically isomorphic to $\ell_{1}^{n}$. A general upper bound

$$
r(X) \leqslant 2-\frac{1}{2+(n-1) 2^{n-1}}
$$

was proved in [1]. The conjecture was finally solved positively by Hinrichs in [6], using properties of the John ellipsoid.

All the previous results are related to real spaces. In [3], the values for some complex spaces are computed. In particular, in that paper it is shown that

$$
r\left(\ell_{1}^{n}(\mathbb{C})\right)=1-\frac{1}{n}+\frac{1}{2 \pi} \int_{0}^{2 \pi}\left|e^{i \theta}-1 / n\right| d \theta
$$

(where $|\cdot|$ denotes the complex modulus). The goal of this article is to show the inequality

$$
r(X) \leqslant r\left(\ell_{1}^{n}(\mathbb{C})\right)
$$

for any $n$-dimensional complex normed space $X$. This will be proved for $n \geqslant 3$, using the same techniques developed in [6], but with more elaborate computations. The inequality should hold for $n=2$, and probably the same computations should work, sharped in a smart way.

For more information about generalisations of Gross's theorem, and some properties of rendezvous numbers of finite dimensional normed spaces, we refer the reader to [5, $\mathbf{9}, \mathbf{1 2}, \mathbf{1 6}, \mathbf{1 8}$ ]. A survey of contributions to this topic is given in [2].

Given a normed space $X$, we denote by $B$ and $S$ its unit ball and its unit sphere respectively By $B_{2}^{n}$ we denote the Euclidean unit ball in $\mathbb{C}^{n}$. 


\section{Previous Results}

We shall prove the following result.

THEOREM 1. Let $X$ be a complex $n$-dimensional normed space, with $n \geqslant 3$. Then $r(X) \leqslant r\left(\ell_{1}^{n}(\mathbb{C})\right)$.

In the proof, we use properties of the John ellipsoid. We recall briefly the properties we shall use (see [11] for proofs).

Given a complex $n$-dimensional normed space $X=\left(\mathbb{C}^{n},\|\cdot\|\right)$, there is a unique ellipsoid of maximal volume contained in $B$. By an affine transformation, we may assume that this ellipsoid is the Euclidean ball $\left\{x \in \mathbb{C}^{n}:|x| \leqslant 1\right\}$. For $x=\left(x_{1}, \ldots x_{n}\right) \in \mathbb{C}^{n}$, $|x|$ will denote the standard Euclidean norm $|x|=\sqrt{(x, x)}$, where $(x, y)$ denotes the complex scalar product. Then there exist $m$ contact points $v_{1}, \ldots v_{m} \in \mathbb{C}^{n}$ and real scalars $c_{1}, \ldots c_{m}>0$ satisfying

$$
\begin{gathered}
\left\|v_{k}\right\|=\left|v_{k}\right|=1 \text { for } k=1, \ldots n ; \quad \sum_{k=1}^{m} c_{k}=n, \\
x=\sum_{k=1}^{m} c_{k}\left(x, v_{k}\right) v_{k}, \text { or equivalently }|x|^{2}=\sum_{k=1}^{m} c_{k}\left|\left(x, v_{k}\right)\right|^{2} \text { for all } x \in X \\
\left|\left(x, v_{k}\right)\right| \leqslant 1 \text { for all } x \in B, \\
\|x\| \leqslant|x| \leqslant \sqrt{n}\|x\| \text { for all } x \in X .
\end{gathered}
$$

In order to prove

$$
r(X) \leqslant r\left(\ell_{1}^{n}(\mathbb{C})\right)=1-\frac{1}{n}+\frac{1}{2 \pi} \int_{0}^{2 \pi}\left|e^{i \theta}-\frac{1}{n}\right| d \theta
$$

we shall need some properties of the function $f(t)=1 /(2 \pi) \int_{0}^{2 \pi}\left|e^{i \theta}-t\right| d \theta$ which we state in the following two lemmas. The first one is just a verification.

Lemma 1. The function $f: \mathbb{R} \rightarrow \mathbb{R}$ is convex, even, 1-Lipschitz and increasing in $[0,+\infty)$. Moreover

$$
f(0)=1, \quad f^{\prime}(0)=0, \quad f^{\prime \prime}(0)=1 / 2 .
$$

Lemma 2. The function $g(t)=1-t+f(t)$ is decreasing in $[0,1]$.

Proof: Let $t_{1}<t_{2}$. The inequality $g\left(t_{1}\right) \geqslant g\left(t_{2}\right)$ is equivalent to $f\left(t_{2}\right)-f\left(t_{1}\right)$ $\leqslant t_{2}-t_{1}$, which is true since $f$ is 1-Lipschitz.

REMARKs. (1) Lemma 1 implies the inequality $r\left(\ell_{1}^{n}\right)<r\left(\ell_{1}^{n}(\mathbb{C})\right)$, since $f(1 / n)$ $>f(0)=1$. Moreover, asymptotically $r\left(\ell_{1}^{n}(\mathbb{C})\right)=r\left(\ell_{1}^{n}\right)+1 /\left(4 n^{2}\right)+o\left(n^{-3}\right)$.

(2) A consequence of Lemma 2 is that the sequence $r\left(\ell_{1}^{n}(\mathbb{C})\right)=g(1 / n)$ is increasing (its limit equals 2). 


\section{Proof of Theorem 1}

Theorem 1 will be deduced from the following inequality:

$$
\frac{1}{n} \sum_{k} c_{k} \frac{1}{2 \pi} \int_{0}^{2 \pi}\left\|v_{i}-e^{i \theta} x\right\| d \theta \leqslant r\left(\ell_{1}^{n}(\mathbb{C})\right)
$$

for any $x \in S$. Fix $x \in S$, and let $r=|x|$. We know that $1 \leqslant r \leqslant \sqrt{n}$. Let $P$ be the ortogonal projection onto $\operatorname{span}_{\mathbb{C}}\{x\}$, namely $P z=r^{-2}(z, x) x$. Let $Q$ be the complementary projection, $Q z=z-P z$. We may assume, changing $v_{k}$ by $e^{-i \alpha_{k}} v_{k}$ if neccesary, that $\left(x, P v_{k}\right)=|x|\left|P v_{k}\right|$. In this case, set

$$
t_{k}=\left(x, P v_{k}\right)=\left(x, v_{k}\right)=|x|\left|P v_{k}\right| \geqslant 0 .
$$

We shall use the following properties of these numbers, which can be easily checked (see [6, Lemma 2 and Lemma 6] for proofs).

Lemma 3. For any $k=1, \ldots m, 0 \leqslant t_{k} \leqslant 1$. We also have $\sum_{k=1}^{m} c_{k} t_{k}^{2}=r^{2}$.

Consider $K$ be the convex hull of $B_{2}^{n} \cup\left\{e^{i \theta} x: \theta \in[0,2 \pi]\right\}$. It is clearly a ball in $\mathbb{C}^{n}$, whose norm is given by the expression:

$$
\||z|\|= \begin{cases}|z|, & \text { if }|Q z|^{2} \geqslant\left(r^{2}-1\right)|P z|^{2} \\ \frac{1}{r}\left(|P z|+\sqrt{r^{2}-1}|Q z|\right), & \text { if }|Q z|^{2} \leqslant\left(r^{2}-1\right)|P z|^{2}\end{cases}
$$

This is shown by reducing it to the real case proved in [6]. Since $B_{2}^{n} \subset K \subset B$, we clearly have $\|z\| \leqslant|| z|||\leqslant| z \mid$. Therefore, in order to prove (1), it is enough to prove

$$
\frac{1}{n} \sum_{k} c_{k} \frac{1}{2 \pi} \int_{0}^{2 \pi}\|\| v_{k}-e^{i \theta} x \| d \theta \leqslant r\left(\ell_{1}^{n}(\mathbb{C})\right)
$$

To estimate the above integrals, we have to evaluate the Euclidean norms of $P\left(v_{k}-e^{i \theta} x\right)$ $=P v_{k}-e^{i \theta} x$ and $Q\left(v_{k}-e^{i \theta} x\right)=Q v_{k}$ :

$$
\begin{aligned}
& \left|P\left(v_{k}-e^{i \theta} x\right)\right|^{2}=\left|P v_{k}-e^{i \theta} x\right|^{2}=\frac{t_{k}^{2}}{r^{2}}+r^{2}-2 \Re\left(P v_{k}, e^{i \theta} x\right)=\frac{t_{k}^{2}}{r^{2}}+r^{2}-2 t_{k} \cos \theta \\
& \left|Q\left(v_{k}-e^{i \theta} x\right)\right|^{2}=\left|Q v_{k}\right|^{2}=1-\left|P v_{k}\right|^{2}=1-\frac{t_{k}^{2}}{r^{2}} .
\end{aligned}
$$

We shall use the following two lemmas. Let $\gamma$ be the number $(1+\sqrt{5}) / 2$. 
LEMMA 4 . If $r^{2} \geqslant 2$ then

$$
\left|Q\left(v_{k}-e^{i \theta} x\right)\right|^{2} \leqslant\left(r^{2}-1\right)\left|P\left(v_{k}-e^{i \theta} x\right)\right|^{2}
$$

for any $1 \leqslant k \leqslant m$ and any $\theta \in[0,2 \pi]$.

LEMMA 5. If $r^{2} \geqslant \gamma$ then

$$
\left|Q\left(v_{k}-e^{i \theta} x\right)\right|^{2} \leqslant\left(r^{2}-1\right)\left|P\left(v_{k}-e^{i \theta} x\right)\right|^{2}
$$

for any $1 \leqslant k \leqslant m$ and any $\theta \in[\pi / 2,3 \pi / 2]$.

Accordingly, the proof will be divided into three cases. In the most important one, the first case, computations also work for $n=2$. In the other two cases they are valid only for $n \geqslant 3$, but probably inequality (2) is also true for $n=2$.

CASE 1. $r^{2} \geqslant 2$. Lemma 4 implies

$$
\frac{1}{2 \pi} \int_{0}^{2 \pi}\left\|\left|v_{k}-e^{i \theta} x\right|\right\| d \theta=\frac{1}{2 \pi r} \int_{0}^{2 \pi}\left|P v_{k}-e^{i \theta} x\right| d \theta+\sqrt{1-\frac{1}{r^{2}}} \sqrt{1-\frac{t_{k}^{2}}{r^{2}}}
$$

for any $1 \leqslant k \leqslant m$. The vectors $P v_{k}$ and $e^{i \theta} x$ are in the same 1-dimensional vector space, $\operatorname{span}_{\mathbb{C}}\{x\}$. Hence

$$
\frac{1}{2 \pi r} \int_{0}^{2 \pi}\left|P v_{k}-e^{i \theta} x\right| d \theta=\frac{1}{2 \pi} \int_{0}^{2 \pi}\left|\frac{P v_{k}}{r}-e^{i \theta} \frac{x}{r}\right| d \theta=f\left(\left|P v_{k} / r\right|\right)=f\left(t_{k} / r^{2}\right)
$$

Thus

$$
\frac{1}{2 \pi} \int_{0}^{2 \pi}\|\| v_{k}-e^{i \theta} x \| d \theta=h_{r}\left(t_{k}^{2} / r^{4}\right)
$$

where

$$
h_{r}(t)=f(\sqrt{t})+\sqrt{1-\frac{1}{r^{2}}} \sqrt{1-t r^{2}}
$$

The following lemma does the job.

LEMma 6. For $r^{2} \geqslant 2$, the function $h_{r}:\left[0,1 / r^{2}\right] \rightarrow \mathbb{R}$ is concave.

PROOF: We have to show that $h_{r}^{\prime}(t)=1(2 \sqrt{t}) f^{\prime}(\sqrt{t})-\left(r \sqrt{r^{2}-1} / 2 \sqrt{1-t r^{2}}\right)$ is a decreasing function in $\left(0,1 / r^{2}\right)$. Letting $t=s^{2}$, we have to prove that

$$
s \in(0,1 / r) \mapsto \frac{1}{s} f^{\prime}(s)-\frac{r \sqrt{r^{2}-1}}{\sqrt{1-r^{2} s^{2}}}
$$


is decreasing. Differentiation with respect to $s$ and symplification give the following inequality to prove:

$$
s f^{\prime \prime}(s)-f^{\prime}(s) \leqslant r^{3} \sqrt{r^{2}-1} \frac{s^{3}}{\left(1-r^{2} s^{2}\right)^{3 / 2}} \quad 0<s<1 / r<1 / \sqrt{2} .
$$

In order to get this inequality, we shall use repeatedly the following elementary property: given two derivable functions $F, G:[a, b] \rightarrow \mathbb{R}$ such that $F(a)=G(a)$ and $F^{\prime}(x) \leqslant G^{\prime}(x)$ for all $x \in[a, b]$, then $F \leqslant G$ in $[a, b]$.

Both sides of the inequality above are null for $s=0$, so what is left to show is that

$$
f^{\prime \prime \prime}(s) \leqslant 3 r^{3} \sqrt{r^{2}-1} \frac{s}{\left(1-r^{2} s^{2}\right)^{5 / 2}} .
$$

Differentiating under the integral sign gives

$$
f^{\prime \prime \prime}(s)=\frac{-3}{2 \pi} \int_{0}^{2 \pi} \frac{(s+\cos \theta) \sin ^{2} \theta}{\left(1+s^{2}+2 s \cos \theta\right)^{5 / 2}} d \theta
$$

and hence $f^{\prime \prime \prime}(0)=0$. Therefore, both sides of the inequality are equal (null) for $s=0$, and using the property again, we are reduced to proving

$$
f^{i v}(s) \leqslant 3 r^{3} \sqrt{r^{2}-1} \frac{1+4 r^{2} s^{2}}{\left(1-r^{2} s^{2}\right)^{7 / 2}}
$$

The function $r \in(\sqrt{2}, 1 / s) \mapsto 3 r^{3} \sqrt{r^{2}-1}\left(1+4 r^{2} s^{2} /\left(1-r^{2} s^{2}\right)^{7 / 2}\right)$ is increasing, and so the second term in the inequality is greater than or equal to

$$
6 \sqrt{2} \frac{1+8 s^{2}}{\left(1-2 s^{2}\right)^{7 / 2}}
$$

and thus it remains to prove that

$$
f^{i v}(s) \leqslant 6 \sqrt{2} \frac{1+8 s^{2}}{\left(1-2 s^{2}\right)^{7 / 2}}, \quad s \in(0,1 / \sqrt{2}) .
$$

Differentiation again under the integral sign shows that

$$
f^{i v}(s)=\frac{6}{\pi} \int_{0}^{2 \pi} \frac{\sin ^{2} \theta}{\left(1+s^{2}+2 s \cos \theta\right)^{5 / 2}} d \theta-\frac{15}{2 \pi} \int_{0}^{2 \pi} \frac{\sin ^{4} \theta}{\left(1+s^{2}+2 s \cos \theta\right)^{7 / 2}} d \theta .
$$

The second term in the right side of the inequality is negative, so

$$
f^{i v}(s) \leqslant \frac{6}{\pi} \int_{0}^{2 \pi} \frac{\sin ^{2} \theta}{\left(1+s^{2}+2 s \cos \theta\right)^{5 / 2}} d \theta
$$




$$
\begin{aligned}
& =\frac{12}{\pi} \int_{0}^{\pi} \frac{\sin ^{2} \theta}{\left(1+s^{2}+2 s \cos \theta\right)^{5 / 2}} d \theta \\
& \leqslant \frac{12}{\pi} \int_{0}^{\pi} \frac{\sin \theta}{\left(1+s^{2}+2 s \cos \theta\right)^{5 / 2}} d \theta \\
& =\frac{8}{\pi} \frac{3+s^{2}}{\left(1-s^{2}\right)^{3}} .
\end{aligned}
$$

Finally, the proof is completed by showing that the latter is less than or equal to

$$
6 \sqrt{2} \frac{1+8 s^{2}}{\left(1-2 s^{2}\right)^{7 / 2}}
$$

To deduce inequality (2), average (3) and use Lemma 6 to obtain

$$
\frac{1}{n} \sum_{k} c_{k} \frac{1}{2 \pi} \int_{0}^{2 \pi}\|\| v_{k}-e^{i \theta} x \| d \theta=\frac{1}{n} \sum_{k} c_{k} h_{r}\left(t_{k}^{2} / r^{4}\right) \leqslant h_{r}\left(\frac{1}{n} \sum_{k} c_{k} t_{k}^{2} / r^{4}\right) .
$$

Using Lemma 3, we have

$$
=h_{r}\left(1 / n r^{2}\right)=f(1 / r \sqrt{n})+\sqrt{1-\frac{1}{r^{2}}} \sqrt{1-\frac{1}{n}} \leqslant f(1 / r \sqrt{n})+1-\frac{1}{r \sqrt{n}}=g(1 / r \sqrt{n}) .
$$

Finally, Lemma 2 and the inequality $r \leqslant \sqrt{n}$ yield

$$
\leqslant g(1 / n)=(1-1 / n)+f(1 / n)=r\left(\ell_{1}^{n}(\mathbb{C})\right)
$$

as desired.

CASE 2. $\gamma \leqslant r^{2}<2$.

By Lemma 5 , for $\theta \in[\pi / 2,3 \pi / 2]$,

$$
\begin{aligned}
\left\|v_{k}-e^{i \theta} x\right\| \| & =\frac{1}{r}\left[\left|P v_{k}-e^{i \theta} x\right|+\sqrt{r^{2}-1}\left|Q v_{k}\right|\right] \\
& =\frac{1}{r} \sqrt{r^{2}+\frac{t_{k}^{2}}{r^{2}}-2 t_{k} \cos \theta}+\sqrt{1-\frac{1}{r^{2}}} \sqrt{1-\frac{t_{k}^{2}}{r^{2}}}
\end{aligned}
$$

In an other case,

$$
\left\|\left|v_{k}-e^{i \theta} x\right|\right\| \leqslant\left|v_{k}-e^{i \theta} x\right|=\sqrt{1+r^{2}-2 t_{k} \cos \theta}
$$


Set $I=[\pi / 2,3 \pi / 2]$ and $I^{c}=[0,2 \pi] \backslash I$. Then

$$
\begin{aligned}
& \frac{1}{2 \pi} \int_{0}^{2 \pi}\left\|v_{k}-e^{i \theta} x\right\| d \theta \\
& \quad \leqslant \frac{1}{2 \pi} \int_{I^{c}} \sqrt{1+r^{2}-2 t_{k} \cos \theta} d \theta+\frac{1}{2 \pi} \int_{I} \sqrt{1+\frac{t_{k}^{2}}{r^{4}}-2 \frac{t_{k}}{r^{2}} \cos \theta} d \theta \\
& \quad=\frac{1}{2 \pi} \int_{I^{c}}\left(\sqrt{1+r^{2}-2 t_{k} \cos \theta}+\sqrt{1-\frac{1}{r^{2}}} \sqrt{1-\frac{t_{k}^{2}}{r^{2}}}\right.
\end{aligned}
$$

Now use $\|\cdot\|_{L^{1}} \leqslant\|\cdot\|_{L^{2}}$ in both integrals for the probability measure $(d \theta) / \pi$ to get

$$
\begin{aligned}
\frac{1}{2}\left(\sqrt{\frac{1}{\pi} \int_{I^{c}}\left(1+r^{2}-2 t_{k} \cos \theta\right) d \theta}+\sqrt{\frac{1}{\pi} \int_{I^{c}}\left(1+\frac{t_{k}^{2}}{r^{4}}+\frac{2 t_{k}}{r^{2}} \cos \theta\right) d \theta}\right) \\
=\frac{1}{2}\left(\sqrt{1+r^{2}-\frac{4 t_{k}}{\pi}}+\sqrt{1+\frac{t_{k}^{2}}{r^{4}}+\frac{4 t_{k}}{\pi r^{2}}}\right)
\end{aligned}
$$

Applying the inequality $\sqrt{a+x} \leqslant \sqrt{a}+(x / 2 \sqrt{a})$ in both square roots we obtain that the latter expression is at most

$$
\frac{\sqrt{1+r^{2}}+1}{2}-\frac{t}{\pi \sqrt{1+r^{2}}}+\frac{t^{2}}{4 r^{4}}+\frac{t}{\pi r^{2}}
$$

The expression above is a convex parabola as a function of $t \in[0,1]$, and so its maximum is attained at one of the extreme points of the interval. For $t=0$, it equals

$$
\frac{\sqrt{1+r^{2}}+1}{2} \leqslant \frac{\sqrt{3}+1}{2}
$$

and for $t=1$,

$$
\frac{\sqrt{1+r^{2}}+1}{2}+\frac{1}{4 r^{4}}-\frac{1}{\pi}\left(\frac{1}{\sqrt{1+r^{2}}}-\frac{1}{r^{2}}\right)
$$

Let $s=r^{2}$. The function

$$
s \in[\gamma, 2] \mapsto \frac{\sqrt{1+s}+1}{2}+\frac{1}{4 s^{2}}-\frac{1}{\pi}\left(\frac{1}{\sqrt{1+s}}-\frac{1}{s}\right)
$$

is convex. Computing at the extreme points of the interval yields

$$
\begin{aligned}
& s=\gamma \mapsto 1.40451 \ldots \\
& s=2 \mapsto 1.4039 \ldots
\end{aligned}
$$


and therefore the maximum is attained at $s=\gamma$, and (4) is (recall that $\gamma^{2}=\gamma+1$ ) $\leqslant \gamma+1 / 2+1 /\left(4 \gamma^{2}\right)$. Hence

$$
\frac{1}{2 \pi} \int_{0}^{2 \pi}\|\| v_{k}-e^{i \theta} x \| d \theta \leqslant \frac{\gamma+1}{2}+\frac{1}{4 \gamma^{2}}+\frac{1}{2} \sqrt{1-\frac{1}{r^{2}}} \sqrt{1-\frac{t_{k}^{2}}{r^{2}}}
$$

Multiplication by $c_{k} / n$, summation over $k=1, \ldots m$, and Cauchy-Schwarz inequality give

$$
\begin{aligned}
\frac{1}{n} \sum_{k=1}^{m} \frac{c_{k}}{2 \pi} \int_{0}^{2 \pi}\left\|v_{k}-e^{i \theta} x\right\| d \theta & \leqslant \frac{\gamma+1}{2}+\frac{1}{4 \gamma^{2}}+\frac{1}{2} \sqrt{1-\frac{1}{r^{2}}} \sqrt{1-\frac{1}{n}} \\
& \leqslant \frac{\gamma+1}{2}+\frac{1}{4 \gamma^{2}}+\frac{1}{2 \sqrt{2}} \sqrt{1-\frac{1}{n}}
\end{aligned}
$$

For $n=3$, the latter is $(\gamma+1) / 2+1 /\left(4 \gamma^{2}\right)+1 /(2 \sqrt{3}) \simeq 1.69318 \ldots<r\left(\ell_{1}^{3}(\mathbb{C})\right)$ $\simeq 1.69464 \ldots$ For $n \geqslant 4$, the bound above is $<(\gamma+1) / 2+1\left(4 \gamma^{2}\right)+1 /(2 \sqrt{2})$ $\simeq 1.75806 \ldots<r\left(\ell_{1}^{4}(\mathbb{C})\right) \simeq 1.76569 \ldots \leqslant r\left(\ell_{1}^{n}(\mathbb{C})\right)$. For $n=2$, the estimate equals to $(\gamma+1) / 2+1 /\left(4 \gamma^{2}\right)+1 / 4 \simeq 1.65451 \ldots$, but $r\left(\ell_{1}^{2}(\mathbb{C})\right) \simeq 1.56354 \ldots$, so this calculation does not prove (2) in the case $n=2$.

The only point remaining is the convexity of the function given by (5). Differentiating twice, we get

$$
\begin{aligned}
& -(1+s)^{-3 / 2} / 8+\frac{3}{2} s^{-4}-\frac{3}{4 \pi}(1+s)^{-5 / 2}+\frac{2}{\pi} s^{-3} \\
& >-\frac{1}{8}(1+\gamma)^{-3 / 2}+\frac{3}{2} 2^{-4}-\frac{3}{4 \pi}(1+\gamma)^{-5 / 2}+\frac{2}{\pi} 2^{-3} \simeq 0.20187 \ldots>0 .
\end{aligned}
$$

CASE 3. $1 \leqslant r^{2} \leqslant \gamma$.

The inequality ||$|\cdot||| \leqslant|\cdot|$ gives

$$
\frac{1}{2 \pi} \int_{0}^{2 \pi}\|\| v_{k}-e^{i \theta} x||\left|d \theta \leqslant \frac{1}{2 \pi} \int_{0}^{2 \pi}\right| v_{k}-e^{i \theta} x \mid d \theta=\frac{1}{2 \pi} \int_{0}^{2 \pi} \sqrt{1+r^{2}-2 t_{k} \cos \theta} d \theta .
$$

Finally, apply again the inequality $\|\cdot\|_{L^{1}} \leqslant\|\cdot\|_{L^{2}}$ for the probability measure $(1 / 2 \pi) d \theta$ to obtain

$$
\leqslant\left(\frac{1}{2 \pi} \int_{0}^{2 \pi}\left(1+r^{2}-2 t_{k} \cos \theta\right) d \theta\right)^{1 / 2}=\sqrt{1+r^{2}} \leqslant \sqrt{1+\gamma}=\gamma .
$$

Since $\gamma \simeq 1.61803 \ldots$ and $r\left(\ell_{1}^{3}(\mathbb{C})\right) \simeq 1.69464 \ldots$, we have the inequality $\gamma<r\left(\ell_{1}^{n}(\mathbb{C})\right)$ for $n \geqslant 3$. Again the argument does not work for $n=2$. 


\section{REFERENCES}

[1] M. Baronti, E. Casini and P. L. Papini, 'On the average distance property and the size of the unit sphere', Atti Sem. Mat. Fis. Univ. Modena, 46 (1998), 427-446.

[2] J. Cleary, S. A. Morris and D. Yost, 'Numerical geometry-numbers for shapes', Amer. Math. Monthly 93 (1986), 260-275.

[3] J.C. García-Vázquez and R. Villa, 'The average distance property of the spaces $\ell_{\infty}^{n}(\mathbb{C})$ and $\ell_{1}^{n}(\mathbb{C})$ ', Arch. Math. 76 (2001), 222-230.

[4] O. Gross, 'The rendezvous value of a metric space', Ann. of Math. Stud. 52 (1964), 49-53.

[5] A. Hinrichs, 'The average distance property of classical Banach spaces', Bull. Austral. Math. Soc. 62 (2000), 119-134.

[6] A. Hinrichs, 'Averaging distances in finite dimensional normed spaces and John's ellipsoid', Proc: Amer. Math. Soc. 130 (2001), 579-584.

[7] P-K. Lin, 'The average distance property of Banach spaces', Arch. Math. 68 (1997), 496-502.

[8] S.A. Morris and P. Nickolas, 'On the average distance property of compact connected metric spaces', Arch. Math. 40 (1983), 459-463.

[9] P. Nickolas and D. Yost, 'The average distance property for subsets of Euclidean space', Arch. Math. 50 (1988), 380-384.

[10] W. Stadje, 'A property of compact connected spaces', Arch. Math. 36 (1981), 275-280.

[11] N. Tomczak-Jaegermann, Banach-Mazur distances and finite dimensional operator ideals, Pitman Monographs and Surveys in Pure and Applied Mathematics 38 (Longman Scientific \& Technical, Harlow, 1995).

[12] C. Thomassen, 'The rendezvous number of a symmetric matrix and a compact connected metric space', Amer. Math. Monthly 107 (2000), 163-166.

[13] R. Wolf, 'On the average distance property of spheres in Banach spaces', Arch. Math. 62 (1994), 338-344.

[14] R. Wolf, 'On the average distance property in finite dimensional real Banach spaces', Bull. Austral. Math. Soc. 51 (1994), 87-101.

[15] R. Wolf, 'Averaging distances in certain Banach spaces', Bull. Austral. Mat. Soc. 55 (1997), 147-160.

[16] R. Wolf, 'On the average distance property and certain energy integrals', Ark. Mat. 35 (1997), 387-400.

[17] R. Wolf, 'Averaging distances in real quasihypermetric Banach spaces of finite dimension', Israel J. Math. 110 (1999), 125-151.

[18] R. Wolf, 'On the existence of certain measures appearing in distance geometry', Arch. Mat. 76 (2001), 308-313.

Facultad de Matemáticas

Universidad de Sevilla

Apdo. 1160, Sevilla 41080

Spain

e-mail: garcia@us.es

villa@us.es 